\title{
Editorial
}

William A. Suk* and Mathuros Ruchirawat

\section{Challenges in children's environmental health in the Asia-Pacific region}

https://doi.org/10.1515/reveh-2019-0080

Children's environmental health (CEH) is an issue that has gained increased attention internationally due to the fact that children are an important vulnerable population to both communicable and non-communicable diseases (NCDs). The first International Conference on Environmental Threats to the Health of Children: Hazards and Vulnerability organized by the World Health Organization in 2002 in Bangkok, Thailand, served as a basis to garner support and research in CEH globally. Since this large international meeting, environmental health scientists, public health professionals, and pediatricians in Southeast Asia and the Western Pacific have launched significant efforts to build research programs, prevention programs, and communication programs to reduce threats to CEH caused by environmental exposures (1).

Although progress has been made to understand the susceptibility to environmental exposures that children face in the Asia-Pacific region, several challenges remain. Deaths among children under the age of 5 years worldwide has decreased by almost 40\%; however, the majority of deaths in children in that category occur in low- to middle-income countries, with Asia and Africa bearing a disproportionate burden (2). An important and newly emerging research challenge is determining the developmental stage-specific exposure and window of susceptibility that children can face, from in utero exposures to exposures during childhood. Researchers are attempting to elucidate the mechanisms responsible for susceptibility and the consequences a developing fetus or child faces with adverse exposures in the critical development stages that can lead to disease either from birth, starting in early childhood, or even remaining latent until presenting itself in adulthood. Significant gaps lie in these research realms and are imperative to explore further to understand at what age and level of development a child is most vulnerable to environmental exposures.

An ever-growing challenge is how to combat the emerging concerns and persistent problems in the region. As the region develops, there are epidemics of NCDs in children, as well as the persistent problems of various infectious diseases. The Asia-Pacific region is not only dealing with malaria, measles, and diarrheal diseases, but they are now faced with rapidly increasing rates of asthma, diabetes, neurodevelopmental disorders, learning disorders, cancer, and other NCDs. Both morbidity and mortality from these diseases have been projected to increase dramatically over the next few decades (3). The predominant risk factor for the NCDs is pollution in the lower- and middle-income countries, whereas pollution and behavior risk factors are of equal importance in NCD causation in the upper-middle-income countries in the Asia-Pacific region (4). Researchers and public health practitioners in the region are faced with the reality of prioritizing and planning for multiple threats to children's health, a task that even highly developed countries struggle to accomplish.

An additional challenge faced in $\mathrm{CEH}$ is the translation from basic discoveries to actual prevention or intervention programs that can prevent disease and improve health for children in the Asia-Pacific region. Translation of research results is often difficult, due to the need to tailor the message in a way that is culturally appropriate and easy for the audience to understand. This issue poses an even more significant difficulty in the Asia-Pacific region because of the large numbers of ethnic groups, languages spoken, and cultural customs practiced. Translating the science in a broad-enough manner for these various ethnic groups to relate to, understand, and accept will continue to be a major challenge and obstacle for researchers and public health practitioners to tackle. The translation of the research also requires strong public health leadership at a national level, which can be a challenge in the region due to competing priorities.

For the translation of research to happen, there needs to be solid research findings that have been vetted in a peer-reviewed process. As is the case in every region, there can be no research message to translate if the findings are not there. More research needs to be conducted in the Asia-Pacific region to understand the fundamental, environmental, disease progression axis as it relates to children. The focus should be placed on research being fundamental in nature, looking at early developmental 
exposures with an emphasis on disease endpoints in children and adolescents, which should also be solution oriented. This type of research will aid in the understanding of gene-product, gene-environment, and gene-vectorenvironment interactions. A challenge in this area is that scientists have a tendency to look at their favorite chemical or exposure, and they miss the salient point that there can be multiple chemicals or exposures that can lead to disease. Although more challenging, starting at the disease as the endpoint and working back from the disease may be the solution. The use of state-of-the-art informatics and tools in research are a way to overcome some of the research challenges; however, some countries in the Asia-Pacific region may not have access to these tools and techniques.

With many topic areas competing for attention in the Asia-Pacific region, environmental health and $\mathrm{CEH}$ may not rise to the top as priorities. However, chronic, NCDs, many of which may be caused by environmental pollution, are the leading cause of death in the AsiaPacific region and are projected to increase even further. By prioritizing diseases caused by environmental pollution and developing a comprehensive agenda to understand the most dangerous environmental exposures in children in order to prevent them, the health and well-being of many can be improved in the Asia-Pacific region.

\section{References}

1. Suk WA, Sly PD. Ensuring a bright future for children's environmental health. Ann Global Health. 2016;82(1):1-2.

2. Suk WA, Ruchirawat M, Stein R, Diaz-Barriga F, Carpenter D, Neira $M$, et al. Health consequences of environmental exposures in early life: coping with a changing world in the post-MDG era. Ann Global Health 2016;82(1):20-7.

3. Yiengprugsawan V, Healy J, Kendig H. Health system responses to population ageing and noncommunicable diseases in Asia. Manila: World Health Organization, Regional Office for the Western Pacific, 2016.

4. Landrigan PJ, Fuller R, Hu H, Caravanos J, Cropper M, Hanrahan D, et al. Pollution and global health - an agenda for prevention. Environ Health Perspectives 2018;126(8):084501.

*Corresponding author: William A. Suk, National Institute of Environmental Health Sciences, Research Triangle Park, NC, USA, E-mail:suk@niehs.nih.gov

Mathuros Ruchirawat: Chulabhorn Research Institute, Bangkok, Thailand 